\title{
Vitamin D status is a predictor of telomere length during pregnancy
}

\author{
M. S. Mulhern ${ }^{1}$, K. Broberg ${ }^{2}$, A. McAfee ${ }^{1}$, E. Laird ${ }^{1}$, H. Li ${ }^{2}$, J. M. W. Wallace ${ }^{1}$, E. M. McSorley ${ }^{1}$, \\ G. J. Myers ${ }^{3}$, P. W. Davidson ${ }^{3}$, S. W. Thurston ${ }^{3}$, C. F. Shamlaye ${ }^{4}$, Ge Watson ${ }^{3}$, E. van Wijngaarden ${ }^{3}$ \\ and J. J. Strain ${ }^{1}$ \\ ${ }^{1}$ Northern Ireland Centre for Food \& Health (NICHE), University of Ulster, Coleraine, BT52 ISA, \\ ${ }^{2}$ Division of Occupational and Environmental Medicine, Lund University, Sweden, ${ }^{3}$ University of Rochester, \\ School of Medicine and Dentistry, United States and ${ }^{4}$ Ministry of Health, Seychelles
}

Telomeres are the protective cap on the end of eukaryotic chromosomes and are essential regulators of cellular lifespan ${ }^{(1)}$. Telomere length (TL) shortens with each DNA replication, reducing the integrity and stability of the chromosome. TL attrition accelerates with inflammation and oxidative stress, and studies have shown shorter TL in individuals with vascular and autoimmune diseases ${ }^{(2)}$. In addition to its effects on bone health, hypovitaminosis D has also been implicated in the development of a number of these diseases ${ }^{(3)}$. Vitamin D is known to have anti-inflammatory effects and thus, may play a role in reducing damage associated with inflammation and oxidative stress $^{(4)}$. It has recently been reported that vitamin D status is positively associated with leukocyte TL in women ${ }^{(5)}$ and supplementation with $50 \mu \mathrm{g}$ vitamin D/d has been shown to increase the activity of telomerase, the enzyme involved in telomere lengthening ${ }^{(6)}$.

The aim of this study was to investigate associations between maternal vitamin D status and maternal and child TL in a population whose vitamin D status should be expected to be optimal, owing to high UVB exposure from the sun, the main source of vitamin D.

This study was conducted as part of the Seychelles Child Development and Nutrition Study. Blood samples were taken from pregnant women at 28 weeks gestation $(n=130)$ and from their children at 5 years of age $(n=117)$. Maternal vitamin D status $(25$-hydroxyvitamin $\mathrm{D}(25(\mathrm{OH}) \mathrm{D})$ concentration) was measured in serum by LC-MS. Relative TL was determined in whole blood samples from both mothers and children using quantitative polymerase chain reaction. Spearman correlation analysis was used to test associations between maternal vitamin D status and TL of mothers and children.

\begin{tabular}{|c|c|c|c|c|c|}
\hline & $\mathrm{N}$ & Mean & SD & Minimum & Maximum \\
\hline Maternal age (yr) & 130 & 26.9 & 5.8 & 17.0 & 40.3 \\
\hline Maternal 25(OH)D (nmol/L) & 130 & 104.6 & 50.0 & 40.2 & 297.0 \\
\hline Maternal TL & 130 & 0.640 & 0.111 & 0.401 & 0.984 \\
\hline Child TL at $5 \mathrm{yr}$ & 117 & 0.706 & 0.098 & 0.446 & 0.918 \\
\hline
\end{tabular}

As expected, mean vitamin D status of the women at 28 weeks gestation was considered optimal, even according to higher cut-off levels for sufficiency $(25(\mathrm{OH}) \mathrm{D}>75 \mathrm{nmol} / \mathrm{l})$. Mothers vitamin D status was significantly correlated with maternal TL $(r=0.260, P=0.003)$ but not with child TL $(r=-0.066, P=0.479)$. Maternal TL was not correlated with child TL. As age is known to negatively influence both $25(\mathrm{OH}) \mathrm{D}$ concentration and TL, albeit not in this cohort, the data were analysed separately, split by mean maternal age. Mothers $25(\mathrm{OH}) \mathrm{D}$ concentration at 28 weeks gestation was positively correlated with maternal TL, but only in the older age group $(r=0.111, P=0.391-v s-$ $r=0.387, P=0.001$, younger $-v s$ - older age group respectively).

These data add to the emerging body of evidence linking vitamin D and TL. The immunomodulatory effects of vitamin D may be one mechanism by which it is protective against telomere attrition and warrants further investigation, including the possible confounding effects of age.

This work was supported by grants from the US National Institute of Environmental Health Sciences, National Institutes of Health, the Government of Seychelles and by the EU through its Sixth Framework Programme.

1. Raynaud CM, Sabatier L, Philipot O et al. (2008) Crit Rev Oncol Hematol 66(2): 99-117.

2. Fitzpatrick AL, Kronmal RA, Gardner JP et al. (2007) Am J Epidemiol 165(1): 14-21.

3. Moreno LA, Valtuena J, Perez-Lopez F et al. (2011) Ann Nutr Metab 59(1): 22-7.

4. Di Rosa M, Malaguarnera M, Nicoletti et al. (2011) Immunology 134(2): 123-39.

5. Richards JB, Valdes AM, Gardner JP et al. (2007) Am J Clin Nutr 86(5): 1420-5.

6. Zhu H, Guo D, Li K et al. (2011) Int J Obes. 